\title{
Medicine Delivery Drone
}

\author{
${ }^{1}$ Pragati Jain, ${ }^{1}$ Ashutosh Rai, \\ ${ }^{1}$ Bobby Budhwani \\ ${ }^{1}$ Student
}

Dept. of E\&Tc, Bharati Vidyapeeth

(Deemed to be University), College of Engineering, Pune, India

\author{
${ }^{2}$ Prof. Sudhir Kadam \\ ${ }^{2}$ Professor \\ Dept. of E\&Tc, Bharati Vidyapeeth \\ (Deemed to be University), College of Engineering, \\ Pune, India
}

\begin{abstract}
In this study we are implementing a medicine drone delivery system using CC3D flight controller which is configured using Open pilot Ground Control Station software. The drone is optimized to deliver the medicine in rural areas, its location can be tracked using GPS together with the Blynk software. The location tracker have been carried out using NodeMCU. This drone can be of great use in certain regions where the geographical terrain is uneven and not fit for proper transportation, also in the areas where there is still lack of proper transportation services available.
\end{abstract}

Keywords- Drone,F450,Open Pilot Ground Control Station(GCS) software, GPS, CC3D, Ardupilot, Blynk, FS-CT6B

\section{INTRODUCTION}

Drones, a common term in this modern technologically advanced \& growing world has been a great invention and has been proven of great use in the last decade. We have seen its implementation in different areas like the military organizations in order to patrol dangerous areas to monitor for any potential threat or illegitimate activities. It is of great use when it comes to provide efficient and convenient surveillance. We have seen drones applications in farming equipment where it is used to spread medicines in the organic fields, carry out easy and safe pesticides disposal over the crops. In the same manner we have developed a drone which is able to deliver essential and vital medicines in the areas where proper transportation is not available for the native people. This drone is capable of delivering the medicine to the places where it cannot be transported using any mechanical vehicle commonly used by all delivery agencies. In any pandemic situation where human interaction are not advised and social distancing is the key in health management structure, this Unmanned drone medicine delivery system can be used as a strong weapon to fight against the pandemic. In emergency situations where the present transportation structure is shattered due to flood earthquake etc. this type of automatic drone delivery system can save precious lives with much less and nominal efforts.

Not only in the rural areas but also in fully developed cities this project can be of great use. The developed and fast moving cities are majorly bothered with slow moving traffic jams. In India, there are many cases reported where the late delivery of medicines to any health organization proved to be very fatal, therefore this drone delivery project will come handy in case of efficient delivery of medicine in the cities where traffic congestion and bad conditions of road become a major drawback in all the above mentioned situations. The increasing population and increasing private transport will result in heavy traffic on the city roads therefore a better air transport system must be developed and this project is one small efforts towards this objective.

All these problems can be solved by the device which we have developed. Inefficient transportation of medicine and other health related problems which were present due to poor delivery procedures can be overcome using this device. The GPS system programmed in this device using node MCU provides accurate locations of the flying drone so one can see and get an idea that where the drone is and can figure out the expected delivery time accordingly. The Blynk app used to build the interface between the device and mobile screen. This is much faster than the traditional motor delivery system.

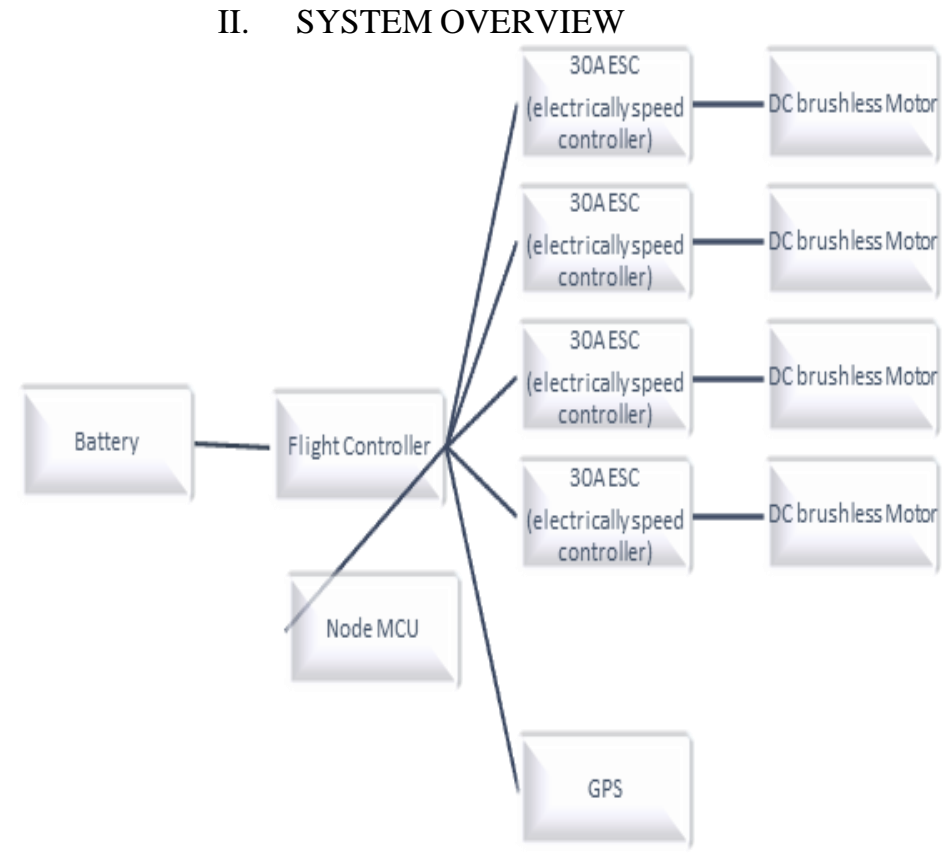

Fig. 2.1. Block Diagram of the System

\section{A. Quadcopter}

Quadcopter is referred to a drone that is controlled by four rotors. In this quadcopter we have used F450 frame as the base. Microcontroller used in this quadcopter is CC3D Flight controller. The intelligence of the drone is the Flight controller. In our project we have made a manually operated Quadcopter which is controlled by a remote. The remote using radio signals sends the signals to radio receiver connected to the controller. Flight controller on receiving these signals controls and regulates the rate of speed of DC brushless motors by providing signals to the ESC. The motors used plays a vital role in deciding the payload of drone. The motors used provides a maximum payload of $1200 \mathrm{kV}$ which is 
sufficient for the purpose of carrying medicines. We have attached a box to carry medicines in our drone.

\section{B. Live Position Tracking}

In our project we have used global positioning system in order to determine the accurate geographical location of the drone to navigate drone containing time sensitive items such as medicines to the delivery address.

\section{WORKING}

The complete working of this system can be divided in different parts which are as follows:

\section{A. Flight Controller}

The brain of drone is flight controller. In this project we have used a CC3D Flight Controller. It is a powerful 32 bit microcontroller with $128 \mathrm{~kb}$ flash and $20 \mathrm{~KB}$ RAM. We have configured controller using user friendly Open Pilot Ground Control Station software (GCS). This controller controls the speed of DC brushless motors by providing signals to the electronic speed controller.

\section{B. Electronic Speed Controller}

Electronic speed controller is a circuit used for regulating and controlling speed of motor. Electronic speed controller generates 3 phase AC power supply to run the electric motors. It is a trapezoidal wave generator. It generates 3 separate waves one for each wire connecting the motor. We have used 30 A electronic speed controller. Controlling speed of $\mathrm{dc}$ brushless motors by changing the timing pulses of current delivered to the windings of motor. We have used 4 electronic speed controllers for controlling 4 DC brushless motors of Quadcopter.

\section{Brushless DC Motors}

A quadcopter requires 4 DC brushless motors. The speed of these motors is controlled and regulated by 4 electronic speed controllers. The speed of motor is regulated by adjusting the frequency of 3 phase electric signals. These motor runs on dc electric Source produced by switching power supply, that produces an AC signal which drives motor. The rotor part of motors is made of several electric windings.

\section{Global Positioning System}

To determine the accurate geographical location of drone GPS is used. We have used an Ublox NEO 6M GPS Module. We have used GPS to track location of the delivery drone and with the help of this it became easy to navigate the drone containing medicines to the remote locations and to the addresses where the medicines are to be delivered.

\section{E. Node $M C U$}

In this drone we used Node MCU to connect mobile phone with the global positioning system to determine the accurate geographical location of the drone. To track the live location of drone and for greater accuracy we have used a Node MCU.

\section{F. Propeller}

Propellers are devices that transform rotatory motion into linear thrust. Drone propellers provide lift for the drone by spinning and developing an airflow which results in pressure difference the top and bottom surfaces of the propeller. Spinning of the drone is controlled by using clockwise propellers and counter clockwise propellers. This eliminates the reactive torque and keeps the drone stable. The general configuration of Quadcopter drone is two clockwise propellers diagonal to each other and two counter clockwise propellers diagonal to each other.

\section{G. Battery}

Battery provides power to the drone. We have used rechargeable lithium polymer battery ( $\mathrm{LiPo}$ ) of capacity rating $2200 \mathrm{mAH}$. Lithium polymer battery have high energy density in relation to their size and weight, with a higher voltage per cell of approximate 3.7 volts.

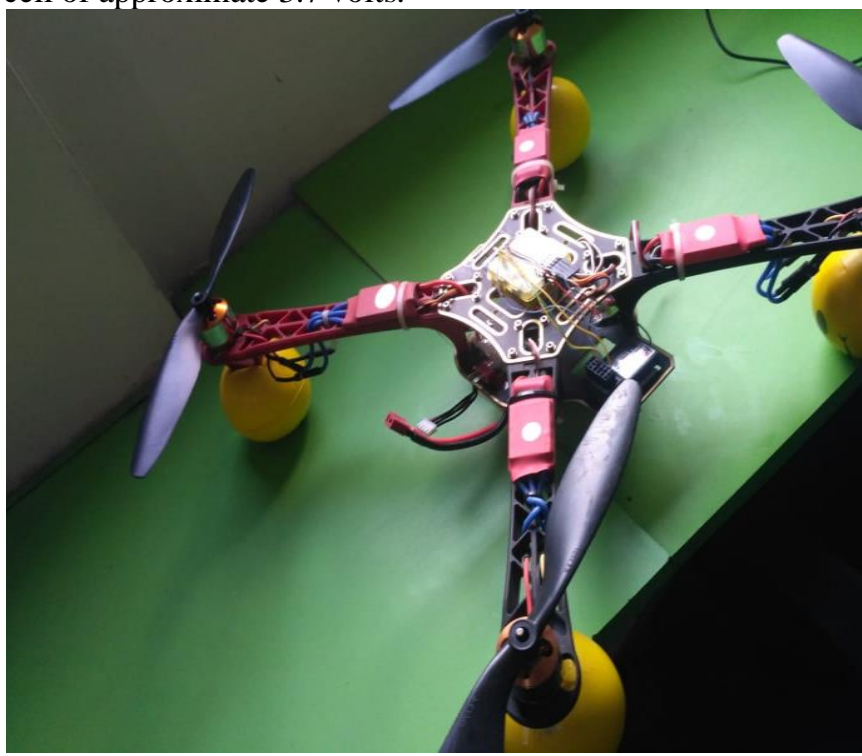

Fig. 3.1.

\section{H. Remote}

Remote is an electronic device that uses radio signals to transmit commands wirelessly to the radio receiver. We have used FS-CT6B remote. It is configured by using T6 config software. Remote sends signals to the FS-R6B receiver connected to the flight controller. Remote is powered with a $12 \mathrm{v}$ battery power supply.

\section{Blynk Mobile Application}

Blynk is an open source IOT platform, can control hardware remotely, can display sensor data, can store data and visualize it. Here in our case to sense the live location of drone we have interface NodeMCU and GPS to Blynk.

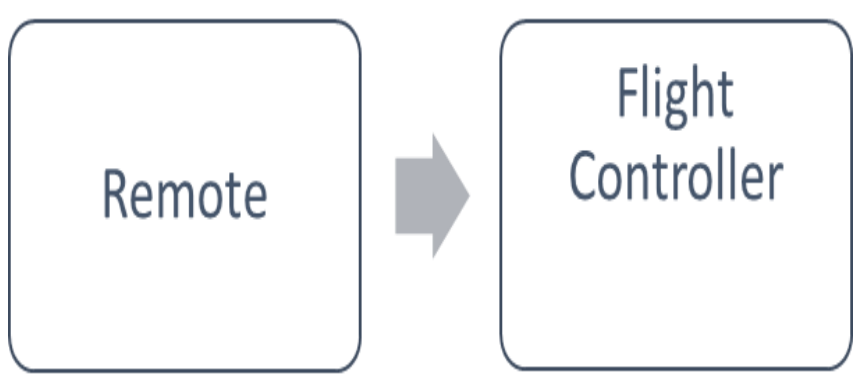




\section{FUTURE SCOPE}

In our project we have used a manually operated drone to deliver medicines to the desired delivery address. Today many companies are researching on developing automated drones that can deliver medicines to remote locations automatically without the help of man. These types of automated drones will be very helpful in delivering time sensitive items such medicines to patients at their door step. This will be very beneficial in providing contactless delivery to the patients in pandemic situation. Drone technology is very popular today but it can be enhanced to various applications in future.

\section{CONCLUSION}

This project is helpful in providing necessary medicines in areas where normal traffic transportations services are not \& also in regions where the geographical terrain is not fit for traditional transportation methods.

Secondly, crucial applications comes in emergency situations like floods earthquake etc. where the resident and doctors need vital medicines which can be delivered easily via our medicine drone delivery system.

Thirdly, this Drone comes handy in cities also. The rising population and tremendous increase in private vehicles on city roads have increased traffic congestions making it difficult for the traditional delivery systems to function effectively. Hence this drone finds its application their also.

\section{ACKNOWLEDGMENT}

This project has been possible because of the hard work and sincere efforts of not only the students but also the project guide, who helped in making the ideas clearer and also provided the necessary information relevant to the project topic.

We want to show our gratitude towards Prof. Sudhir Kadam sir in helping us through the project, his continuous motivation and pertinent counselling helped us to achieve our goals.

We would like to show our gratitude towards our institution, Bharati Vidyapeeth(DU)College of Engineering, for giving us this opportunity to work in this project and polish our skills in order to improve our knowledge.

Lastly a sincere thanks to the Department of Electronics and Telecommunication for giving us platforms to present our ideas and also improvising all along.

\section{REFERENCES}

[1] Towards Automated Drone Surveillance in Railways: State-of-theArt and Future Direction Conference Paper . October 2016 from book Content-Based Mammogram Retrieval Using Mixed Kernel PCA and Curvelet Transform (pp.336-348).

[2] Unmanned Aerial Vehicle (DRONE) Priyanka Kumari, Isha Raghunath, Akanksha Mishra, Pinkey Sharma, Ankita Pandey, Mr Sachchidanand Jaiswal Department of Electronic and Communication Engineering, Buddha Institute of Technology, Uttar Pradesh, India

[3] Surveillance and Security Management System Using Drone Ganeshwar.R, Ramprabhu.J, Manochitra.P, Kaliappan.S, Department of EEE, Kumaraguru College of Technology, Coimbatore.

[4] International Journal of Engineering Sciences and Research Technology Implementation of Voice Activated Autonomous QuadcopterPritesh A. Metha, M.E. (E\&TC) \& Prof. M. U. Inamdar Siddhant, College of Engineering, Pune. 